Tema: Solidificação / Lingotamento

\title{
CONTRIBUIÇÃO PARA A CARACTERIZAÇÃO E DIMINUIÇÃO DA OCORRÊNCIA DE DEFEITOS INTERNOS DO TIPO TRINCA OFF-CORNER EM BARRAS LAMINADAS*
}

\author{
Érica Castilhos ${ }^{1}$ \\ Jair Janoski ${ }^{2}$ \\ Paulo Carvalho Fernandes ${ }^{3}$ \\ Telmo Strohaecker
}

\begin{abstract}
Resumo
Defeitos internos do tipo trinca off-corner são um dos principais problemas relacionados à qualidade em tarugos de aços especiais lingotados continuamente. Este tipo de defeito é bastante crítico, pois além de se apresentar de forma descontínua no tarugo e, consequentemente na barra laminada, não é detectável pelos equipamentos de inspeção em linha usualmente utilizados para garantir a qualidade final do material. Neste trabalho, foram avaliadas amostras de barras laminadas de um aço baixo carbono com e sem a presença do defeito do tipo trinca off-corner a fim de estabelecer um método que permita avaliar a criticidade da trinca. Estes resultados foram relacionados com os principais fatores que influenciam na formação deste defeito, proporcionando alterações de processo com o objetivo de minimizar a sua ocorrência.
\end{abstract}

Palavras-chave: Trinca off-corner; Lingotamento Contínuo; Caracterização de Defeito.

\section{CONTRIBUTION TO THE CHARACTERIZATION AND OCCURRENCE MINIMIZATION OF OFF-CORNER CRACKS IN ROLLED BARS}

\begin{abstract}
Off-corner internal cracks are one of the major problems related to the quality of special steel billets continuously casted. This defect is quite critical since it is discontinuous and it is not detectable by the inspection lines. In this work samples of low carbon steel rolled bars with and without the presence of off-corner cracks were evaluated in order to create a method to assess the crack criticality. These results were correlated with the main factors which have some influence on the formation of this defect by providing some process modifications in order to minimize their occurrence.
\end{abstract}

Keywords: Off-corner cracks; Continuous casting; Characterization of defects.

1 Engenheira Metalúrgica, Mestranda PPGEM, UFRGS, Engenheira de Processos, Gerdau, Usina Charqueadas, Charqueadas, RS, Brasil.

Técnico em Metalurgia, Analista Técnico, Gerdau, Usina Charqueadas, Charqueadas, RS, Brasil. Engenheiro Metalúrgico, M.Sc.,Consultor Técnico, Gerdau Aços Especiais Brasil, Charqueadas, RS, Brasil.

4 Engenheiro Metalúrgico, Professor Doutor, Departamento de Metalurgia e PPGE3M, UFRGS, Porto Alegre, RS, Brasil.

* Contribuição técnica ao 450 Seminário de Aciaria - Internacional, 25 a 28 de maio de 2014, 


\section{INTRODUÇÃo}

Um dos maiores problemas encontrados durante o lingotamento contínuo de aços especiais é a formação de defeitos como trincas internas e superficiais ${ }^{1}$. Nos últimos anos, muitos trabalhos têm sido desenvolvidos buscando-se aumentar a qualidade dos aços visto que estas trincas podem ser transferidas para os produtos finais, gerar grandes sucateamentos internos ou, caso não sejam detectados, transferidos para o cliente gerando grande insatisfação ${ }^{1}$. Assim surge a necessidade de se conhecer as possíveis causas e contramedidas necessárias para o seu controle e caracterização.

A identificação destes defeitos posteriormente ao processo de lingotamento contínuo, geralmente é realizada através de análise específica (análise de Baumann ou macrografia) de uma seção transversal do tarugo. Por serem realizadas de forma amostral, estas análises não são suficientes para garantir a completa identificação e eliminação dos tarugos defeituosos previamente à etapa de laminação. Desta forma, após o processo de laminação, geralmente realiza-se, uma etapa de inspeção em linha para identificação de trincas superficiais e internas presentes nas barras laminadas. Este tipo de inspeção em linha é bastante eficiente para a detecção de trincas superficiais, mas, devido ao fato de que alguns defeitos internos apresentamse preenchidos por óxidos ou sulfetos, como as trincas off-corner, tal inspeção não consegue garantir $100 \%$ de isenção destas descontinuidades ao produto. Assim sendo, torna-se necessário proporcionar ao processo de lingotamento contínuo a maior robustez possível, visando minimizar a incidência destes defeitos.

\subsection{Trincas Off-corner}

Trinca off-corner é um tipo de defeito interno gerado na etapa de solidificação do aço, localizado próximo aos cantos dos tarugos lingotados continuamente. $\mathrm{O}$ mecanismo de formação da trinca off-corner está relacionado com a não uniformidade da espessura da casca solidificada do tarugo do canto para o meio. A casca se torna mais fina à medida que se aproxima do canto do tarugo, o que é explicado pela redução da taxa de extração de calor, devido à formação de um gap maior quando comparado ao encontrado no meio da face do tarugo [1]. Desta forma, qualquer abaulamento da face do tarugo no molde devido à pressão ferrostática se estende não desde os cantos, mas a partir da região off-corner que irá funcionar como uma espécie de "dobradiça" entre a região central do tarugo e o canto. Esta ação de dobramento gera uma tensão de deformação na frente de solidificação que, se exceder a tensão de fratura, irá formar uma trinca como pode ser visto na Figura 1 [2].

* Contribuição técnica ao $45^{\circ}$ Seminário de Aciaria - Internacional, 25 a 28 de maio de 2014, 

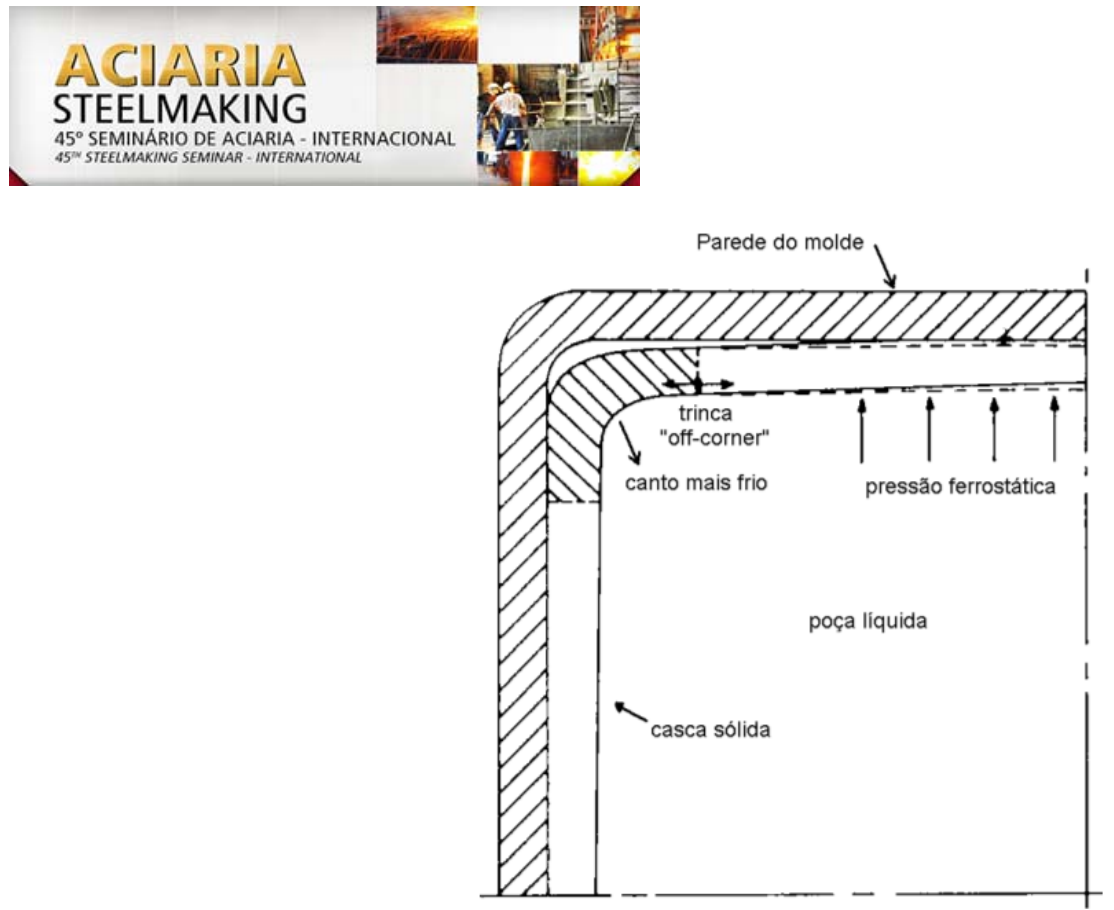

Figura 1. Diagrama esquemático mostrando o abaulamento no molde e a formação de trincas off-corne [3].

\subsection{Fatores que Afetam a Formação de Trincas off-corner}

Diversos fatores ao longo do lingotamento contínuo influenciam na formação de trincas off-corner entre os principais estão a composição química do aço, as condições do molde e os parâmetros que impactam na extração de calor da casca sólida, como a temperatura e velocidade de lingotamento. Em estudo realizado por Ray, et al. [4], observou-se uma alta incidência de trincas off-corner em aços com teores de carbono na faixa de $0,2-0,3 \%$ de carbono. Ainda em relação à composição química, de acordo com Samarasekera [5] para teores de enxofre acima de $0,02 \%$ e relação $\mathrm{Mn} / \mathrm{S}<25$, há um grande aumento na incidência de trincas offcorner. Isto se deve ao fato de que uma baixa relação $\mathrm{Mn} / \mathrm{S}$ leva à formação de um líquido interdendrítico de FeS de baixo ponto de fusão durante a solidificação que favorece a formação de trincas internas e segundo Brimacombe, et al. [6] um aumento no teor de Mn diminui a formação de FeS. Além disso, uma maior relação $\mathrm{Mn} / \mathrm{S}$ é benéfica à qualidade do tarugo, pois melhora a tenacidade da casca solidificada e ajuda a suportar a tensão gerada devido à ação de dobramento causado pelo abaulamento do tarugo [4].

No que diz respeito ao molde, foi visto por Brimacombe [3] que uma conicidade inicial inadequada leva a formação de marcas de oscilação mais profundas favorecendo a formação de trincas off-corner. Já segundo Penna [1], em moldes muito usados, o gap cresce e torna-se maior na parte inferior do molde levando a uma menor extração de calor e em um reaquecimento da superfície na saída do molde. Este reaquecimento pode dar origem a formação de trincas off-corner. Além da conicidade e da vida do molde, o raio de canto causa uma influência na formação de trincas. Em raios de canto menores, o gap entre o molde e a casca sólida é menor, o que irá gerar uma casca sólida mais espessa na região do canto do que na região off-corner, a qual irá possuir uma pele mais fina e frágil podendo levar ao surgimento da trinca [7].

A influência da extração de calor se dá fortemente pela temperatura de lingotamento e pela velocidade. Um aumento na temperatura de lingotamento produz um aumento do comprimento da zona colunar devido ao fato de que em altas temperaturas os cristais equiaxiais formados no molde podem ser mais facilmente refundidos. Este aumento da zona colunar é importante, pois esta é mais susceptível ao trincamento

* Contribuição técnica ao 450 Seminário de Aciaria - Internacional, 25 a 28 de maio de 2014, Porto Alegre, RS, Brasil. 


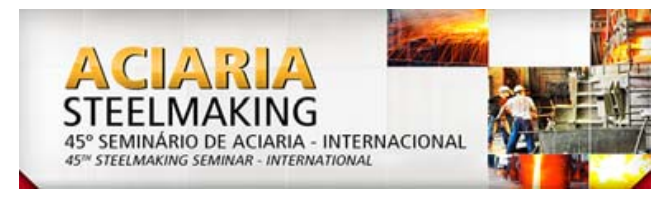

do que a zona equiaxial e, uma zona colunar longa aumenta a severidade da segregação central e porosidade. Além disso, uma alta temperatura de lingotamento leva a uma casca solidificada mais fina e então mais frágil e mais susceptível a formação de trincas off-corner [8]. Lait [8] também observa que a temperatura de lingotamento influencia diretamente a velocidade de solidificação do aço no molde e esta é mais percebida para pequenos tempos de residência, ou seja, em altas velocidades de lingotamento.

O presente trabalho visa estabelecer uma correlação entre os parâmetros que impactam na formação de trincas off-corner a fim de minimizar a ocorrência das mesmas. Além disso, contribuir com um método de caracterização destas trincas em barras laminadas.

\section{MATERIAIS E MÉTODOS}

\subsection{Materiais}

Este trabalho foi realizado utilizando como material de análise barras laminadas redondas com bitolas de até $40 \mathrm{~mm}$ de um aço com faixa de carbono de 0,15\% 0,25\%, considerado para aços construção mecânica como baixo carbono, e baixa relação $\mathrm{Mn} / \mathrm{S}$. De cada ordem de produção deste material, foram retiradas 5 amostras aleatoriamente e analisadas para caracterização do defeito. As amostras foram retiradas de forma aleatória devido a este tipo de defeito não ser detectado na linha de inspeção e, como relatado anteriormente, se apresentar de forma intermitente no tarugo e, posteriormente na barra laminada. A Tabela 1 apresenta as faixas de composição química do aço estudado. Todas as amostras obtidas para este trabalho apresentaram os resultados das análises de composição química dentro das faixas especificadas.

Tabela 1. Faixa de Composição Química do aço analisado

\begin{tabular}{|cccc|}
\hline & C & Mn & S \\
\hline Mín & 0,15 & 0,80 & 0,04 \\
Máx & 0,25 & 1,00 & 0,07 \\
\hline
\end{tabular}

Foram analisadas amostras pertencentes a 4 corridas diferentes, sendo que cada corrida foi dividida em 3 ordens de produção, logo tendo um total de 60 amostras conforme representado na Figura 2. Em todas as análises foi caracterizada a ocorrência ou não do defeito e, quando detectada a presença de trinca off-corner, esta foi caracterizada quanto ao tamanho e profundidade em relação à superfície.

* Contribuição técnica ao $45^{\circ}$ Seminário de Aciaria - Internacional, 25 a 28 de maio de 2014, 

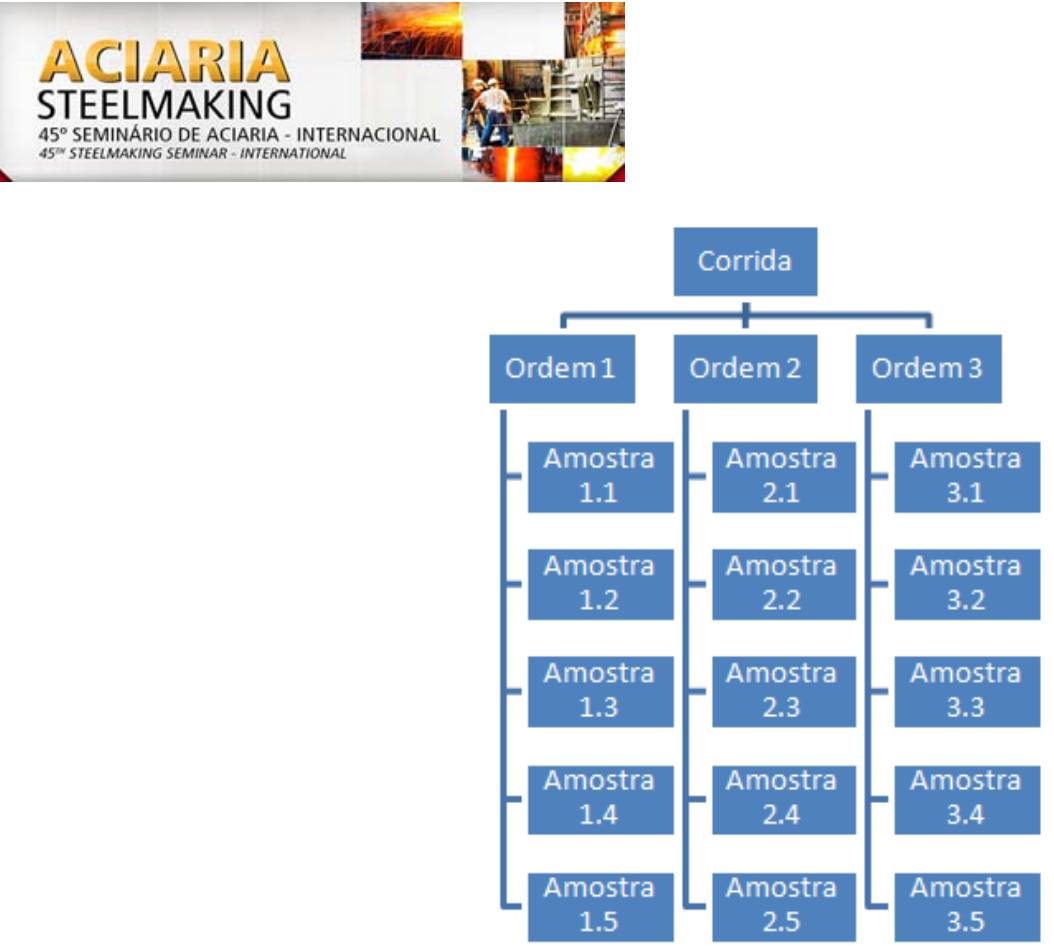

Figura 2. Fluxograma de retirada de amostras para caracterização de defeitos nas barras laminadas.

\subsection{Planejamento de Experimentos}

A fim de garantir uma robustez de processo e a eliminação do defeito do tipo trinca off-corner, foram realizados testes, alterando as variáveis de processo, que se sabe pela literatura ter grande influência na geração deste defeito. Logo, foram realizados testes alterando a relação Mn/S e alterando as condições de refrigeração.

- Relação Mn/S: foi aumentada através do aumento da faixa de Mn que era de 0,8 - 0,9\% para 0,9 - 1,0\%. De acordo com Samarasekera [5], a relação $\mathrm{Mn} / \mathrm{S}$ deve ser superior a 25 para diminuir a susceptibilidade à formação de trincas off-corner. Em função da composição química desta qualidade não permitir valores maiores que 25 , a relação Mn/S foi aumentada apenas para um patamar superior a 20, sendo que anteriormente o patamar era de 15 20.

- Condições de refrigeração: tendo em vista que além da eliminação do defeito, deseja-se manter a produtividade, a velocidade de lingotamento objetivada não foi reduzida, mas atuou-se na modificação do ajuste de velocidade para os casos onde a temperatura de lingotamento (TL) esteja acima da temperatura objetivada devido a variações normais ao processo. Para estes casos, a velocidade de lingotamento é ajustada seguindo uma curva de correção com valores pré-determinados, a fim de manterem-se as condições de solidificação objetivadas. Logo, caso a TL esteja acima do valor objetivado, a velocidade é reduzida e, em casos onde a TL esteja abaixo da objetivada, a velocidade é aumentada. Para os experimentos, foram utilizadas duas curvas de correção de velocidades diferentes. A Curva A, na qual só há atuação na velocidade caso a TL esteja $20^{\circ} \mathrm{C}$ ou mais acima da temperatura objetivada, e a Curva $\mathrm{B}$, na qual há redução de velocidade caso a $T L$ esteja $10^{\circ} \mathrm{C}$ ou mais acima da temperatura objetivada. As curvas de correção de velocidade podem ser vistas na Tabela 2.

* Contribuição técnica ao $45^{\circ}$ Seminário de Aciaria - Internacional, 25 a 28 de maio de 2014, 
Tabela 2. Curvas de correção de velocidade

\begin{tabular}{|cccccc|}
\hline Curva & T obj $+\mathbf{2 0}{ }^{\circ} \mathbf{C}$ & $\mathbf{T ~ o b j ~}+15^{\circ} \mathbf{C}$ & T obj $+10^{\circ} \mathbf{C}$ & T obj $+5^{\circ} \mathbf{C}$ & T obj \\
\hline A & $\begin{array}{c}\text { Correção da } \\
\text { velocidade }\end{array}$ & V obj & V obj & V obj & V obj \\
B & $\begin{array}{c}\text { Correção da } \\
\text { velocidade }\end{array}$ & $\begin{array}{c}\text { Correção da } \\
\text { velocidade }\end{array}$ & $\begin{array}{c}\text { Correção da } \\
\text { velocidade }\end{array}$ & V obj & V obj \\
\hline
\end{tabular}

Com base nestes fatores, foram planejados 4 experimentos de acordo com a técnica estatística denominada DOE (Design of Experiments), a qual utiliza um planejamento fatorial para avaliar a interação entre dois ou mais fatores. Neste tipo de planejamento para cada experimento, todas as possíveis combinações dos níveis dos fatores são avaliadas. No presente estudo, foram estabelecidos dois níveis para cada fator. Desta forma, tem-se um Planejamento Fatorial $2^{k}$, onde $k$ representa 0 número de fatores a serem testados em cada experimento. A Tabela 3 ilustra como estes experimentos foram planejados.

Tabela 3. Planejamento de experimentos

\begin{tabular}{|ccc|}
\hline Experimento & Faixa da relação Mn/S & Curva de correção da velocidade \\
\hline $\mathbf{1}$ & $15-20$ & $\mathrm{~A}$ \\
$\mathbf{2}$ & $15-20$ & $\mathrm{~B}$ \\
$\mathbf{3}$ & $20-25$ & $\mathrm{~A}$ \\
$\mathbf{4}$ & $20-25$ & $\mathrm{~B}$ \\
\hline
\end{tabular}

\subsection{Caracterização de Trincas Off-corner}

A metodologia usada para a caracterização de trincas off-corner neste trabalho é composta pelas seguintes etapas:

- Impressão de Baumann em amostras de tarugo;

- Análise macrográfica de amostras de barra laminada;

- Análise micrográfica de amostras de barra laminada;

- Ensaio de compressão a quente.

\subsubsection{Impressão de Baumann no tarugo}

O ensaio para avaliação de trincas off-corner no tarugo foi realizado seguindo metodologia padronizada em procedimento operacional em conformidade com norma ASTM E 1180 [9]. Para o presente trabalho, foram retiradas três amostras por corrida sendo uma amostra de cada veio de lingotamento para a realização da Impressão de Baumann.

\subsubsection{Análise macrográfica na barra laminada}

Após a operação de laminação, foram retiradas amostras e enviadas ao laboratório metalúrgico para caracterização macrográfica da seção transversal seguindo procedimento interno de acordo com norma ASTM E 381 [10].

Após cortadas, as amostras foram atacadas com solução de Persulfato de Amônio $20 \%$ por 10 minutos, lavadas e secadas. Primeiramente, estas amostras foram analisadas em Estereomicroscópio com aumentos variando de 10 - 60 vezes para a identificação da presença de trincas off-corner. Nesta etapa, foram realizadas medições do tamanho da trinca e sua distância em relação à superfície da amostra de barra laminada. Durante a análise macrográfica também foi analisada a forma e distribuição da trinca off-corner, ou seja, se o defeito se apresentava de forma contínua ou intermitente. Para isso utilizou-se três critérios para classificação da

* Contribuição técnica ao 45 Seminário de Aciaria - Internacional, 25 a 28 de maio de 2014, Porto Alegre, RS, Brasil. 
intensidade da trinca como sendo: fraca, média ou forte, conforme a representação esquemática da Figura 3.

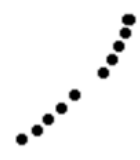

a)

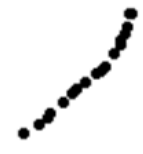

b)

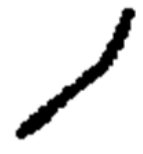

c)

Figura 3. Classificação da intensidade da trinca off-corner de acordo com continuidade do defeito. (a) Fraca, (b) Média e (c) Forte [11].

\subsubsection{Análise micrográfica}

Após a caracterização macrográfica, as amostras foram cortadas nas regiões onde apresentavam trinca off-corner e repreparadas seguindo procedimento metalográfico padrão. As amostras foram atacadas com reagente Nital $2 \%$ e analisadas via microscópio ótico. Nesta etapa, buscou-se mostrar a presença de sulfetos na região da trinca. De acordo com a intensidade da trinca podemos ver estes sulfetos mais ou menos agrupados.

\subsubsection{Ensaio de compressão}

Depois da análise microestrutural, as amostras que apresentaram trincas off-corner foram submetidas ao ensaio de compressão para simular o comportamento do defeito durante forjamento a quente, visto que este material segue este tipo de processamento para atingir sua forma final. O objetivo desta etapa é mostrar a criticidade deste tipo de defeito quando submetido à compressão a quente. Para realização deste ensaio, utilizaram-se amostras com um comprimento de três vezes o raio da barra laminada as quais foram aquecidas em um forno tipo mufla até $980^{\circ} \mathrm{C}$ por uma hora. Depois de aquecidas, as amostras foram comprimidas em uma prensa com capacidade de 250 toneladas com o auxílio de um gabarito até atingir uma vez o raio, ou seja, utilizou-se uma relação de compressão de 3:1.

Desta forma, para cada experimento realizado as amostras foram analisadas e as trincas off-corner encontradas foram classificadas quanto ao tipo, ao tamanho e a distância em relação a superfície das barras laminadas. Após utilizou-se o tamanho da trinca como sendo a variável resposta ao DOE e pode-se desta forma determinar quais os parâmetros de processo mais adequados a fabricação deste material.

\section{RESULTADOS E DISCUSSÃO}

Os experimentos foram realizados em 4 corridas e, para estas, foram medidas as temperaturas de lingotamento durante toda a corrida avaliando como esta se comportava em relação à temperatura objetivada. A atuação na velocidade a fim de corrigir as condições de solidificação nos casos da temperatura estar acima da objetivada ocorreram de acordo com a curva utilizada em cada experimento e os resultados podem ser vistos na Tabela 4.

* Contribuição técnica ao $45^{\circ}$ Seminário de Aciaria - Internacional, 25 a 28 de maio de 2014, 
Tabela 4. Dados reais dos experimentos

\begin{tabular}{|ccccc|}
\hline Experimento & $\begin{array}{c}\text { Relaçã } \\
\mathbf{0} \\
\text { Mn/S }\end{array}$ & $\begin{array}{c}\text { Curva de } \\
\text { correção de } \\
\text { velocidade }\end{array}$ & $\begin{array}{c}\text { Temperatura de } \\
\text { lingotamento }\end{array}$ & $\begin{array}{c}\text { Atuação na } \\
\text { velocidade }\end{array}$ \\
\hline $\mathbf{1}$ & 17,6 & $\mathrm{~A}$ & $\mathrm{~T} \mathrm{obj}+15^{\circ} \mathrm{C}$ & $\mathrm{Não}$ \\
$\mathbf{2}$ & 18,7 & $\mathrm{~B}$ & $\mathrm{~T}$ obj $+15^{\circ} \mathrm{C}$ & $\mathrm{Sim}$ \\
$\mathbf{3}$ & 20,6 & $\mathrm{~A}$ & $\mathrm{~T} \mathrm{obj}+15^{\circ} \mathrm{C}$ & $\mathrm{Não}$ \\
$\mathbf{4}$ & 24,3 & $\mathrm{~B}$ & $\mathrm{~T} \mathrm{obj}+15^{\circ} \mathrm{C}$ & $\mathrm{Sim}$ \\
\hline
\end{tabular}

As análises realizadas indicaram a presença de defeito em $21 \%$ das amostras de barras laminadas e em 33\% das amostras de tarugos sendo que neste último caso, apenas em $17 \%$ das amostras apresentaram defeitos com tamanho acima de 10 $\mathrm{mm}$, considerado muito crítico. Na Tabela 5, é apresentada a distribuição de defeitos nas análises de Baumann.

Tabela 5. Resultado das análises de Baumann (tarugo)

\begin{tabular}{|ccc|}
\hline Experimento & $\begin{array}{c}\text { Max comprimento do defeito } \\
(\mathbf{m m})\end{array}$ & $\begin{array}{c}\text { Distância em relação à superfície } \\
(\mathbf{m m})\end{array}$ \\
\hline $\mathbf{1}$ & 12 & 18 \\
$\mathbf{2}$ & 7 & 13,5 \\
$\mathbf{3}$ & 18 & 11,2 \\
$\mathbf{4}$ & isento & isento \\
\hline
\end{tabular}

As análises realizadas nas barras laminadas abrangeram 60 amostras, sendo 15 amostras para cada experimento, e devido ao grande número de amostras, serão detalhados os resultados para o defeito mais crítico encontrado em cada experimento. Primeiramente, as trincas off-corner encontradas foram classificadas quanto ao número e ao tipo (forte, média, fraca). Após, avaliou-se o tamanho e a distância em relação à superfície. Todas as amostras foram submetidas ao ensaio de compressão para avaliar a abertura do defeito quando submetido a operações de forjamento a quente e estes resultados podem ser vistos na Tabela 6.

Tabela 6. Resultado das análises de metalográficas (barras laminadas)

\begin{tabular}{|cccccc|}
\hline Experimento & $\begin{array}{c}\text { Quantidade } \\
\text { de TOC } \\
\text { encontradas }\end{array}$ & $\begin{array}{c}\text { Classificaçã } \\
\text { o da TOC }\end{array}$ & $\begin{array}{c}\text { Comprimento } \\
\text { do defeito } \\
\text { (mm) }\end{array}$ & $\begin{array}{c}\text { Distância } \\
\text { em relação } \\
\text { à superfície } \\
\text { (mm) }\end{array}$ & $\begin{array}{c}\text { Abertura durante } \\
\text { ensaio de } \\
\text { compressão }\end{array}$ \\
\hline $\mathbf{1}$ & 10 & Forte & 3,8 & 0,9 & Sim \\
$\mathbf{2}$ & 1 & Fraca & 2,2 & 1,7 & Não \\
$\mathbf{3}$ & 4 & Forte & 3,7 & 0,2 & Sim \\
$\mathbf{4}$ & 0 & Isento & - & - & Não \\
\hline
\end{tabular}

Comparando os resultados das análises de Baumann com a presença dos defeitos nas barras laminadas, tem-se que as piores condições de trincas encontradas foram nas corridas que já haviam sido indicadas como críticas nas análises de tarugo. Os piores defeitos encontrados tanto nos tarugos quanto nas barras laminadas foram nos experimentos que utilizaram a curva de correção da velocidade $A$, ou seja, em corridas onde não houve o ajuste da velocidade de lingotamento em função da temperatura estar acima da objetivada, o que mostra a grande influência das condições de solidificação na incidência de trincas off-corner.

As análises do DOE realizadas com o auxilio do software Minitab mostram que a curva de correção da velocidade possui uma maior influência no tamanho da trinca do que a relação $\mathrm{Mn} / \mathrm{S}$. Esse comportamento ocorre devido à máxima relação obtida

* Contribuição técnica ao 450 Seminário de Aciaria - Internacional, 25 a 28 de maio de 2014, Porto Alegre, RS, Brasil. 
já estar abaixo do valor ótimo informado pela literatura, que deve ser maior do que $25^{5}$ (Figura 4). Além disso, pode ser visto pela Figura 5 que quando foi utilizada a curva de correção de velocidade $A$, mesmo para valores de relação $M n / S$ na faixa de 20-25, foi observada a ocorrência de trincas maiores do que as encontradas quando se utilizou a curva de correção de velocidade B.

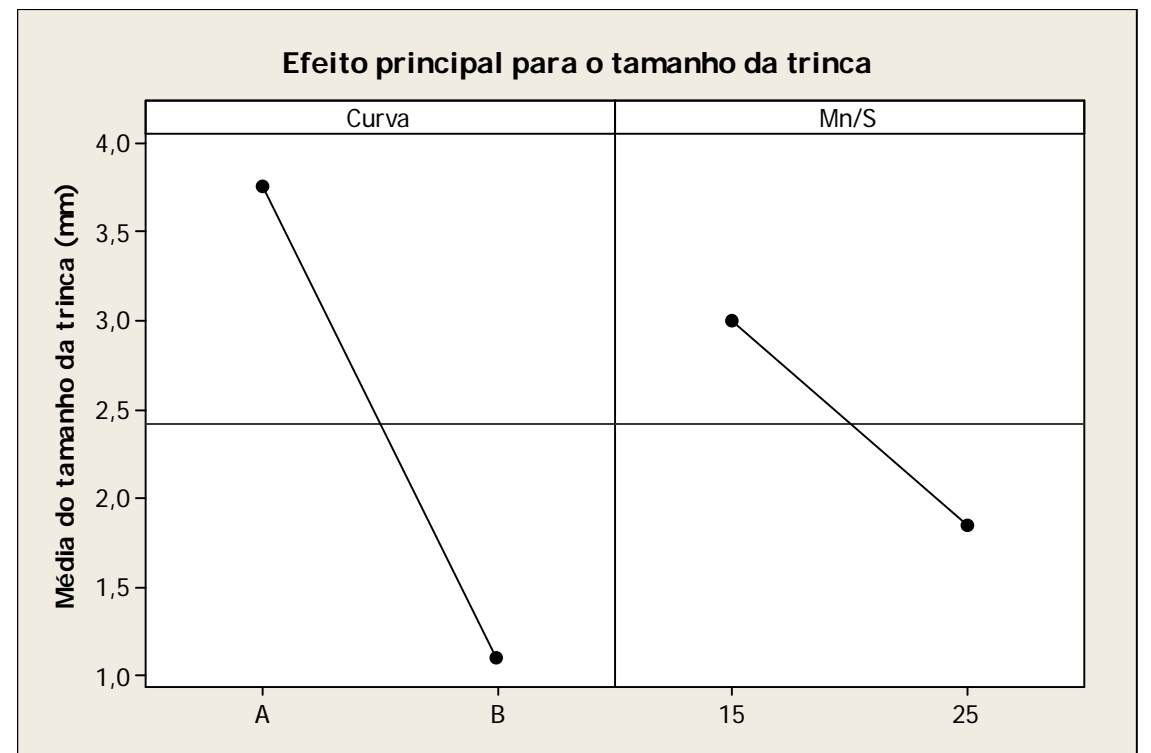

Figura 4. Gráfico do efeito principal para o tamanho da trinca $(\mathrm{mm})$.

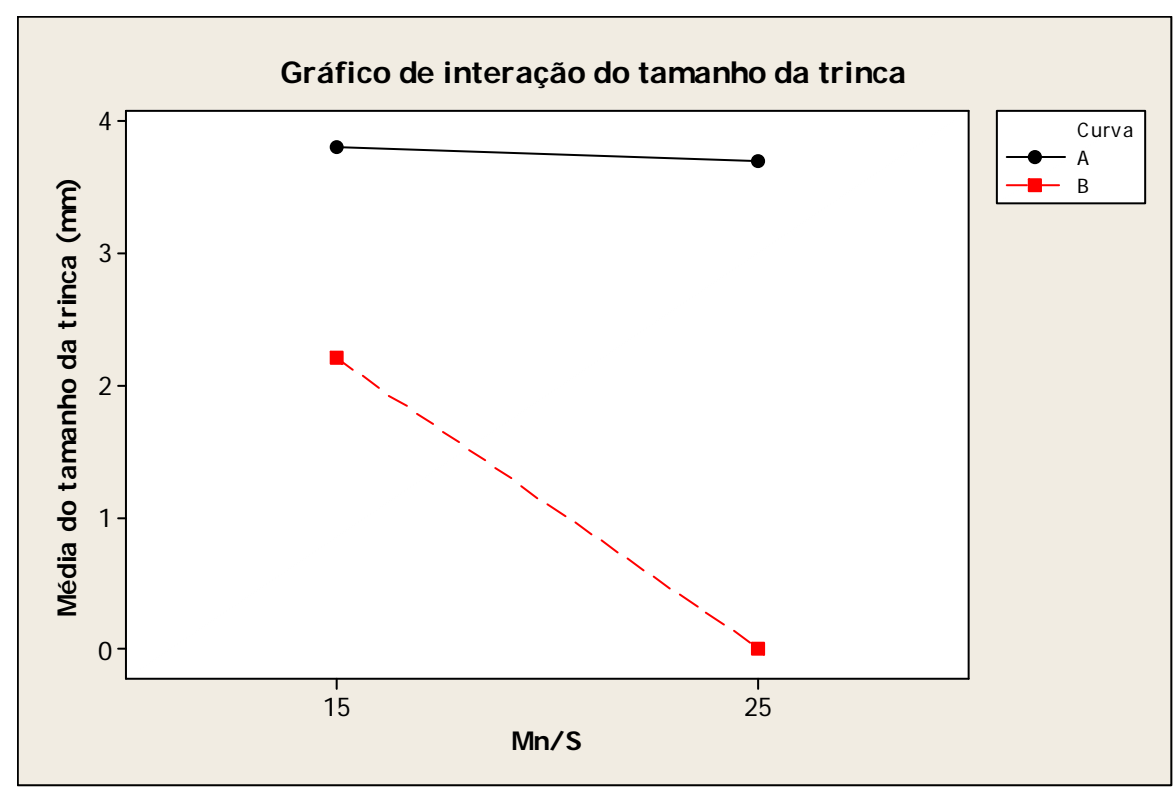

Figura 5. Gráfico de interação para o tamanho da trinca (mm).

Da Figura 6 à Figura 7 é mostrada a sequência de análises realizadas desde a macrografia até a análise via microscopia eletrônica para o experimento 3.

* Contribuição técnica ao $45^{\circ}$ Seminário de Aciaria - Internacional, 25 a 28 de maio de 2014, 

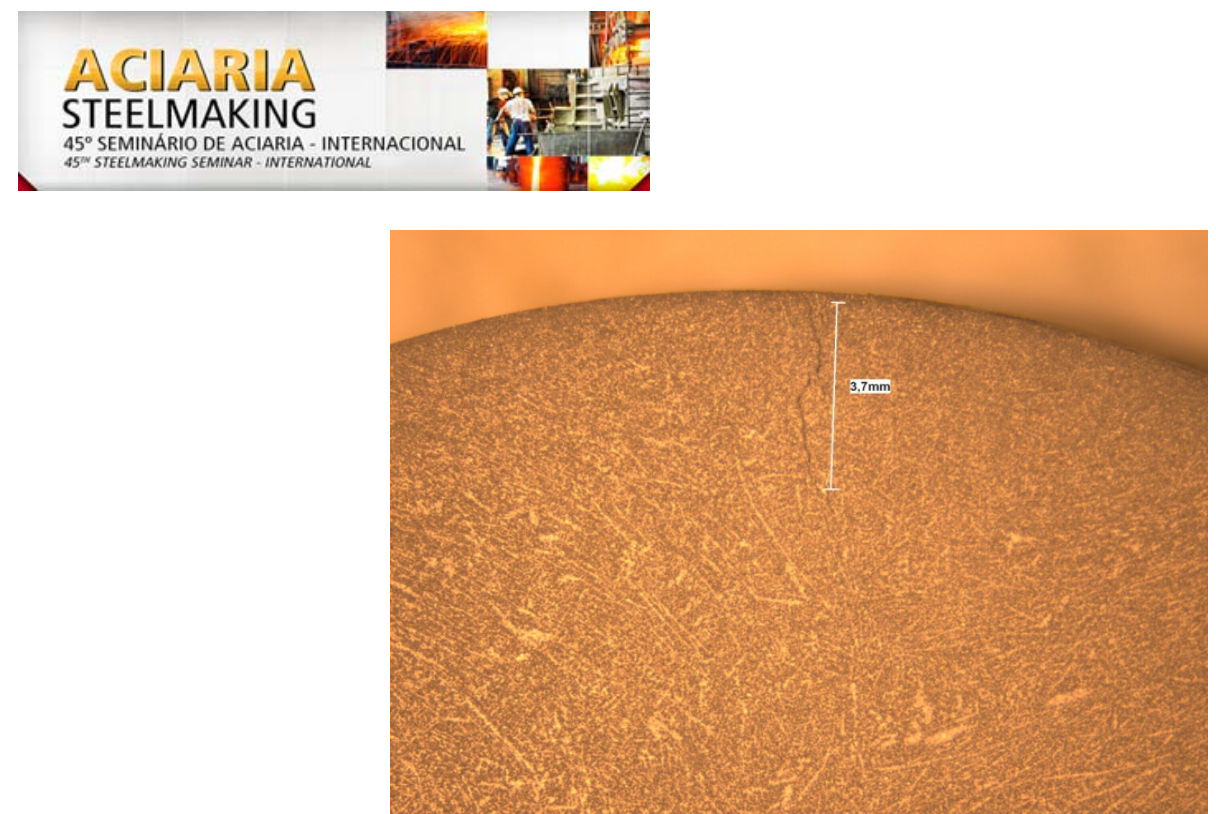

Figura 6. Macrografia mostrando a presença do defeito. Aumento de 10x.

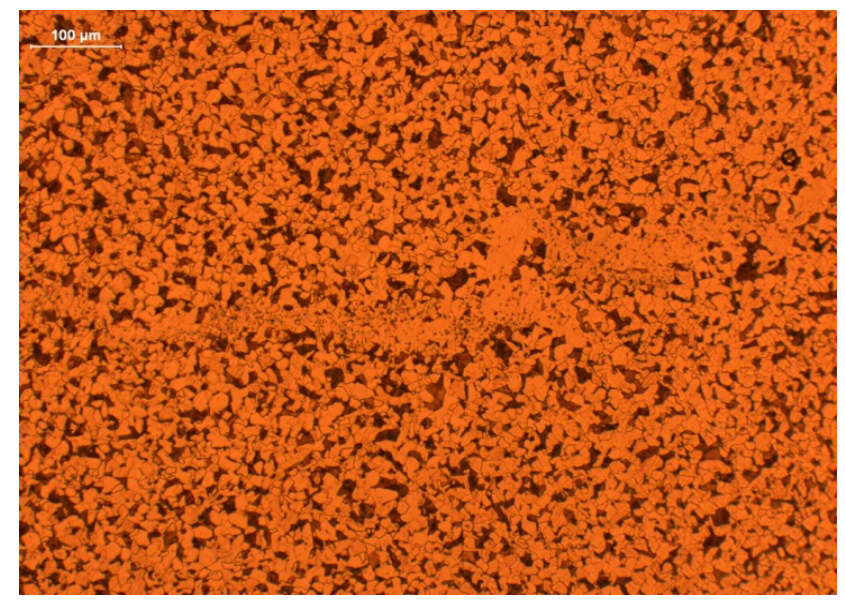

Figura 7. Micrografia, mostrando a presença de Sulfeto no local do defeito. Aumento de 100x.

Na Figura 8 pode ser vista a amostra após o ensaio de compressão a quente, mostrando a abertura no local da trinca. As amostras nas quais houve abertura do defeito durante o ensaio de compressão a quente foram as que apresentaram o maior tamanho da trinca e a distância em relação à superfície menor que $1,0 \mathrm{~mm}$. Estas amostras foram provenientes do experimento 1 e 3 os quais utilizaram a curva de correção de velocidade $A$, confirmando desta forma a resposta da análise do DOE como sendo este o fator mais crítico para a geração do defeito.

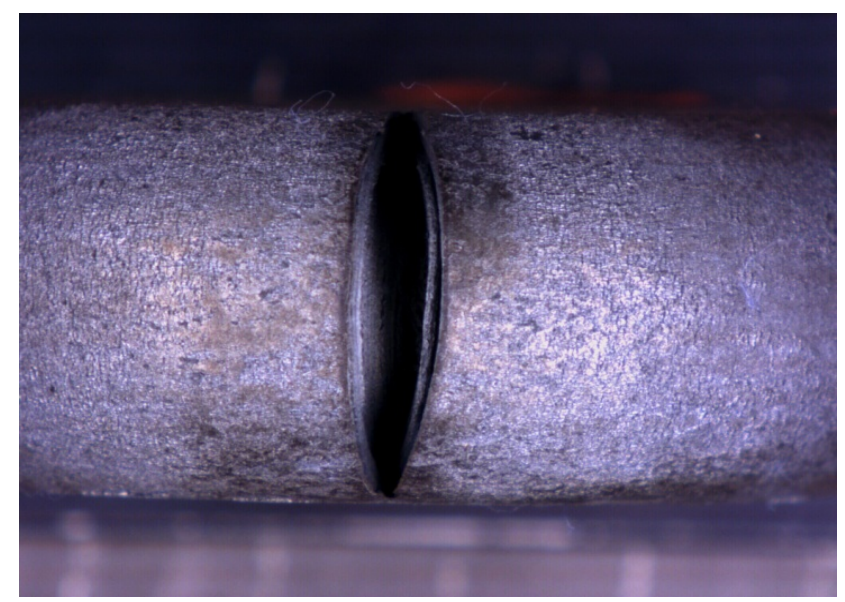

Figura 8. Imagem da amostra após o ensaio de compressão a quente, mostrando que houve a abertura no local da trinca.

* Contribuição técnica ao $45^{\circ}$ Seminário de Aciaria - Internacional, 25 a 28 de maio de 2014, Porto Alegre, RS, Brasil. 
Após estas análises foram definidas as condições do experimento 4 como parâmetros de processo de fabricação deste material, ou seja, relação $\mathrm{Mn} / \mathrm{S}$ entre 20-25 e uso da curva de correção de velocidade B a fim de garantir a eliminação do defeito do tipo trinca off-corner.

\section{CONCLUSÃO}

Com base nos resultados obtidos, foi observado que a curva de correção de velocidade utilizada têm forte influência na incidência de trincas off-corner, assim como a relação $\mathrm{Mn} / \mathrm{S}$. O uso da curva de correção de velocidade A levou a geração de defeitos maiores nas barras laminadas. Logo, através das análises realizadas, definiram-se as condições de relação Mn/S de 20-25 e uso da curva de correção de velocidade $B$ como sendo as ideais para minimização da ocorrência de trincas offcorner neste material.

\section{Agradecimentos}

Os autores agradecem a Gerdau Aços Especiais Brasil pela liberação da divulgação deste trabalho. Agradecem o empenho de toda equipe da Aciaria e do Laboratório Metalúrgico que contribuíram para o desenvolvimento do presente trabalho.

\section{REFERÊNCIAS}

1 Penna LV. Trinca de solidificação off-corner no lingotamento contínuo de tarugos. Belo Horizonte: Escola de Engenharia, UFMG; 2005.

2 Bommaraju R, Brimacombe JK, Samarasekera IV. Mould behaviour and solidification in the continuous casting of steel billets. ISS Transactions. 1984;5:95-105.

3 Brimacombe JK, Hawbolt EBE, Weinberg F. Formation of off-corner internal cracks in continuously-cast billets. Canadian Mettalurgical Quaterly. 1980;19:215-227.

4 Ray SKr, Patwari K, Ray AKr. Influence of Mn/S ratio and casting speed on off-corner cracking of billet. Steel Times International. 2006;abril:20-22.

5 Samarasekera IV. Crack formation in the continuous casting of steel. Brimacombe Continuous Casting Course. Vancouver: Brimacombe Continuous Casting Society; 2011.

6 Brimacombe JK, Sorimachi K. Crack formation in the continuous casting of steel. Metallurgical Transactions. 1977;8:489-505.

7 Park JK, Thomas BG, Samarasekera IV. Analysis of thermomechanical behaviour in billet casting with different mould corner radii. Ironmaking and Steelmaking. 2002;29:359-375.

8 Lait JE, Brimacombe JK. Solidification during continuous casting of steel. ISS Transactions. 1982;1:1-12.

9 American Society for Testing and Materials. ASTM E-1180: Standard practice for preparing sulfur prints for macrostrutural evaluation. West Conshohocken;2010.

10 American Society for Testing and Materials. ASTM E-381 Standard method of macroetch testing steel bars, billets, blooms, and forgings. West Conshohocken; 2010.

11 Gerdau. CHA-PR-265-080: Procedimento interno de ensaio macrográfico [documento interno]; 2009.

\footnotetext{
* Contribuição técnica ao 45 Seminário de Aciaria - Internacional, 25 a 28 de maio de 2014, 